by no means solve the problem.

In summary, this volume provides a comprehensive study of the state of the art in this subject. It accurately reflects the uncertainties as well as the progress made. The standard of treatment is somewhat variable, as might be expected with such a large number of contributors, but the editors have done an excellent job in avoiding overlaps and repetition. The price will probably put the book beyond the reach of even the most enthusiastic individual, but it should certainly find its place in libraries.

V. J. Robinson

$V . J$. Robinson is Lecturer in Radiochemistry at the University of Manchester, UK.

This book is almost identical to the author's 1968 dissertation, with the addition of four pages on the paddle-wheel experiment and the deletion of some material on Mary Somerville and W.R. Grove in chapter 6. Much has been written on nineteenth-century physical science during the past decade and as a result historians of science have not only a different view of the development of energy concepts but an enhanced interest in philosophical and sociological questions. Steffens mentions some of the relevant publications in his notes but has made few substantive changes in his text. Perhaps most serious is his failure to take account of John Forrester's important article on the chemical background of Joule's work (Studies in History and Philosophy of Science, 1975). Ten of the 22 items in Forrester's "Joule bibliography" are missing from Steffens' bibliography (users of the latter should be warned of a mixup of lines of type on pages 165-66).

It is necessary to criticise two features which this book shares with an increasing number of scholarly monographs. First, if notes must (because of the deterioration of printing technology) be placed at the end of the book instead of the foot of the page, they should be keyed to the text pages so one does not have to find out what chapter one is reading in order to locate its notes. Second, the index should cover the notes as well as the text; in this case it doesn't even cover the text adequately (for example, Aepinus has no entry).

Stephen G. Brush abolished the Ptolemaic system; not Newton when he annihilated the Cartesian vortices; no Young and Fresnel, when they exploded the Corpuscular Theory; not Faraday and Maxwell, in their splendid victory over Actio in distans more thoroughly shattered a malignant and dangerous heresy, than did Joule when he overthrew the baleful giant FORCE, and firmly established, by lawful means, the beneficient rule of the rightful monarch, ENERGY! Then, and not till then, were the marvellous achievements of Sadi Carnot rendered fully available; and Science silently underwent a revolution more swift and more tremendous than ever befell a nation".

None of this Victorian enthusiasm for the Manchester brewer's son is conveyed by Henry Steffens, nor does he attempt to explain the significance of the change from "force" to "energy" in physics. He has compiled instead a sober survey of Joule's experiments and theories from 1837 to 1852, together with a discussion of closely related contributions of J.R. Mayer, William Thomson, W.J.M. Rankine, R. Clausius and H. von Helmholtz. Steffens elucidates the path from early experiments on the "electromagnetic engine" to determinations of the mechanical equivalent of heat and formulations of the first law of thermodynamics. Although it provides a clear and accurate account of one aspect of the history of energy conservation, the book suffers from its limited scope. We learn little about Joule's personality or his influence beyond the small circle of scientists mentioned above, and scarcely anything about the concept of energy apart from Joule's rather narrow interpretation of it.

Steffens' most interesting chapter is the one dealing with Joule's rough translation, in his notebook, of Mayer's 1842 paper; he argues that Joule knew of Mayer's work before 1844 and was thereby led to perform the experiments on rarefaction and condensation of air that yielded his third value for the mechanical equivalent of heat. But aside from this, Steffens makes very little use of documents, even those already published (for example, by A.L. Hatton and L. Rosenfeld in 1956, and by J.T. Lloyd in 1970).

\section{Liquid crystalline materials}

Liquid Crystals and Biological Structures. By G.H. Brown and J.J. Wolken. Pp. 187. London, 1979.) \$19; £12.35.

IT is surprising that many distinguished scientists, including Bernal in 1933 and Needham in 1950, should have pointed out so early the importance of the liquid crystalline state for biological systems. Yet it was only in the early 1960 s that a major attempt was made to examine experimentally the liquid crystalline properties of biological molecules. Nowadays when so many scientists have contributed to the study of phospholipid-water systems to provide insight into biomembrane structure and function, it is interesting to remember that a great deal of the basic fundamental work - for example, phase transitions, phase diagrams, fluidity, bile salts and cholesterol effects - was carried out as recently as the $1960 \mathrm{~s}$. When we further note that myelin figures were first observed by Virchow in 1854 it is interesting to consider the socio-economic technological factors which held up the study for so long of these important liquid crystalline lipid-water systems.

Brown and Wolken remind us of this in the present book. They examine the basic properties of liquid crystalline materials and include a discussion of the various classifications, including nematic, cholesteric and smectic liquid crystals. Thermotropic and lyotropic liquid crystal and the neat soap phase important to the (Academic: New York, San Francisco and
Stephen G. Brush teaches history of science at the University of Maryland, College Park. detergent industry is shown. The optical properties of liquid crystals are also adequately but briefly described. These chapters I found neat, and useful. However, the later chapters dealing with the relationship of these fundamental properties of matter to the biological structures are less satisfactory.

The authors include chapters on the cell, photoreceptor structures (that is, chlorophotoreceptor structures, that is, chloroplasts and the eye, fibrous protein structures and effectors, and biomembranes. They also include applications to medicine, such as temperature scanning and diseases. I found these biological chapters a little too superficial for my taste. I would have preferred some better examples of how important an understanding of liquid crystals has been for their biological implications - for example, the way in which thermotropic phase transitions which occur in pure phospholipid-water systems are clearly observed in some natural biomembranes. The use of liposomes as potential pharmacological capsules and the formation of gall-stones are also given only passing reference, and yet these are interesting, useful and important applications of the study of liquid crystalline substances to biology and medicine.

As an overview providing a stimulus for a student or young research worker the book may be valuable. It is clearly written with good diagrams and printing. I observed only a few obvious typographical errors.

Dennis Chapman

Dennis Chapman is Professor of Biophysical Chemistry at the Royal Free Hospital School of Medicine, University of London, UK. 